\title{
INSTITUCIONES ABIERTAS Y CREACIÓN DE CONFIANZA: EXPERIENCIAS DESDE ESPAÑA
}

\author{
Jorge Resina \\ Universidad Complutense de Madrid, España \\ jresina@ucm.es \\ Cecilia Güemes \\ Universidad Autónoma de Madrid, España \\ cecilia.guemes@uam.es
}

\section{RESUMEN}

En un contexto en el que las instituciones tienen como objetivo impulsar nuevas prácticas democráticas que involucren de forma más activa a la ciudadanía, la gobernanza se presenta como principal paradigma de gestión de lo social, este trabajo reflexiona sobre: 1) el papel de la experimentación y los espacios híbridos como escenario propicio para la co-creación, cooperación y colaboración entre distintos actores; y 2) el potencial de herramientas de gobierno abierto y mecanismos de participación ciudadana para reconstruir la confianza en las administraciones. A partir de este enfoque, se analizan tres experiencias innovadoras del ámbito local y autonómico en Espańa, que tienen como fin crear un laboratorio de políticas (Aragón), luchar contra la corrupción (Valencia) y promover el diálogo público (Madrid).

Palabras clave: Confianza, Innovación democrática, Instituciones abiertas, Participación ciudadana, Espańa. 


\title{
OPEN INSTITUTIONS AND TRUST BUILDING: \\ EXPERIENCES IN SPAIN
}

\begin{abstract}
In a context in which institutions seek to promote new democratic practices that involve citizens more actively, governance is presented as the main paradigm of social management and this paper reflects on: 1) the role of experimentation and hybrid spaces as a scenario propitious for co-creation, cooperation and collaboration between different actors; and 2) the potential of open government tools and citizen participation mechanisms to rebuild trust in administrations. Based on this approach, the study analyzes three innovative experiences at the local and regional levels in Spain whose purpose is to create a policy laboratory (Aragón), combat corruption (Valencia) and promote public dialogue (Madrid).
\end{abstract}

Keywords: Trust, Democratic innovation, Open institutions, Citizen participation, Spain. 


\section{INTRODUCCIÓN: LA URGENCIA POR RECONECTAR CON LA CIUDADANÍA}

¿Cómo se concreta la innovación democrática y la gobernanza social en sociedades cada vez más complejas y cambiantes como las actuales? ¿Cuál debe ser el formato que asuman estas nuevas experiencias, para al mismo, tiempo responder a su entorno y transformar el interior de las propias instituciones? ¿En qué medida estos espacios de deliberación y participación son capaces de desarrollar confianzas mutuas entre funcionarios públicos, decisores políticos y ciudadanos? ¿Es la experimentación posible y, sobre todo, recomendable ante desafíos tan sensibles como urgentes?

La falta de confianza hacia las instituciones ha abierto el debate sobre la necesidad de buscar nuevas formas de involucrar a la ciudadanía en los asuntos públicos (Accetti, Mulieri et al. 2015, Waylen 2015, Della Porta 2013). Una búsqueda de soluciones que pasa tanto por una mayor inclusión y participación de actores, como por mejorar la respuesta institucional, con políticas eficaces que resuelvan los problemas reales de las personas (Coleman, Pzybylska y Sintomer 2015, Font, Wojcieszak y Navarro 2015).

Para afrontar este desafío, la administración pública necesita reconfigurar su organización, flexibilizar los modelos de gestión y dirección y transformar el rol y la actitud de los funcionarios, con el fin de ser más permeable a la innovación y los aprendizajes que suceden en sus márgenes. Esta administración ha de sustentarse en dos grandes pilares: la confianza y la mediación. Esto es así ya que la autoridad institucional cumple un papel fundamental de coordinación, orienta la función que ha de cumplir cada uno de los actores e integra a las partes, conectando lo fragmentado en base a la comunicación y el diálogo. La creación de confianza demanda un liderazgo en el que el funcionario/mediador estimula la participación y contribuye a generar compromisos mutuos, mediante el habla, la escucha y la gestión del disenso (Ramió 2017, Brugué 2009, 2004).

Este trabajo busca responder a las cuestiones planteadas al inicio desde un punto de vista tanto teórico como práctico. Para ello, en primer lugar, se plantea una reflexión sobre el papel de las instituciones y los retos que supone la puesta en marcha de nuevas pautas de interacción entre decisores políticos, funcionarios públicos y ciudadanía. Con este fin, nos interesa analizar: 1) cómo se institucionaliza/operacionaliza la introducción de estrategias de democracia participativa y principios del gobierno abierto en las estructuras de gobierno regionales y locales; y 2) el modo en que estas innovaciones institucionales buscan reconfigurar la cultura burocrática administrativa y sentar las bases para el desarrollo de confianzas mutuas 
entre funcionarios y ciudadanos.

En segundo lugar, se presentan tres estrategias innovadoras en España que buscan promover nuevas formas de participación (Aragón), transparencia (Valencia) y colaboración (Madrid). La primera de estas experiencias tiene por meta abrir la administración desde dentro, creando para ello un espacio de experimentación e innovación abierta, el Laboratorio de Aragón [Gobierno] Abierto (LAAAB), que favorezca nuevas formas de participación, aprovechando distintos conocimientos para la creación de políticas. El segundo caso, el sistema de transparencia y alertas contra la corrupción seguido por la Generalitat Valenciana, apuesta por el desarrollo de herramientas y tecnologías que permitan transparentar la actividad pública y detectar y alertas sobre prácticas dudosas, con el fin de recuperar la confianza ciudadana. El tercero se centra en Madrid Escucha, un programa del Ayuntamiento de Madrid cuyo objetivo fue poner a dialogar a ciudadanía y empleados públicos, crear confianza y dar respuesta a problemas públicos de la ciudad de forma colaborativa, a través del codiseño de prototipos experimentales.

\section{INNOVACIÓN DEMOCRÁTICA COMO FÓRMULA PARA CREAR CONFIANZA}

Las preocupaciones que planteamos en este trabajo tienen una triple crisis como punto de partida: de la democracia representativa, del modelo jerárquico de gobierno y de la administración pública como institución.

En relación a la democracia, la crisis de representación reclama un debate más allá del componente electoral, orientada a enriquecer la discusión pública y abrir los procesos de toma de decisiones. Este ejercicio de democratización supone avanzar en diferentes direcciones, desde la mejora de la representación entre representantes y representados (democracia representativa) hasta la inclusión de actores tradicionalmente excluidos (democracia inclusiva), pasando por la creación de nuevos espacios de deliberación (democracia deliberativa) y mecanismos de participación directa (democracia directa) (Subirats 2000, Elster 1998, Castoriadis 1996, Young 1990, Máiz 2001).

Si las herramientas de democracia directa, inclusiva y deliberativa son la respuesta a la crisis de la representación, la teoría de la gobernanza y el paradigma del gobierno abierto, lo son a la crisis de gobernabilidad jerárquica como modelo de gestión de lo público. La idea de gobernanza colaborativa tiene en el diálogo y la cooperación su clave organizativa a través de la interdependencia y el intercambio de información. En un contexto 
en el que desafíos como el cambio climático, la seguridad o la creación de empleo se caracterizan por una complejidad creciente, se demandan soluciones multi-actor en las que se aprovechen las distintas capacidades. La probabilidad de encontrar respuestas dependerá, en buena medida, de que se generen estas sinergias y que de ellas surjan procesos de inteligencia colectiva y estrategias de resiliencia y reacción ante lo inesperado (Pollitt, Bouckaert y Löffler 2007, Aguilar Villanueva 2006, Jessop 1996).

Ahora bien, para que democracia y gobernanza puedan realizarse se necesita un modelo de administración pública con disposición al diálogo y plasticidad para probar nuevos diseños organizativos y ser receptiva a su entorno. Desde esta óptica, junto a sus funciones tradicionales de regulación y provisión de bienes y servicios, las organizaciones públicas asumen un rol de "habilitador cívico" que tiene por objetivo reforzar las capacidades de la ciudadanía (Sirianni 2009). Para ello, se requiere de procesos paulatinos de apertura de la administración que generen las condiciones necesarias para la cooperación entre actores y la creación de confianza (Putnam 2001).

Sobre los pilares de gobierno abierto (transparencia, colaboración y participación), se rediseña la institución para, en el orden interno, transformar las organizaciones a nivel funcional, estructural y relacional; y en el externo, incluir a la ciudadanía a formar parte de la toma de decisiones. En ambos casos, se trata de un ejercicio incremental que incluso cuando no cumple con sus expectativas democratizadoras, genera un impacto a modo de aprendizaje (Welp y Ordóńez 2017, Cornwall 2004, Fung y Wright 2001, Cabrero y Arellano 1993).

Tabla 1: Regímenes y modelos de gobierno en crisis y respuestas

Crisis

Democracia representativa

Gobierno jerárquico

Administración pública weberiana
Respuestas

Democracia directa, deliberativa, participativa

Gobernanza social

Gobierno abierto

Fuente: Elaboración propia.

Articuladas las respuestas a las tres crisis, la innovación en la administración se orienta a promover prácticas de creación y diseño compartidas, con las personas como centro del proceso (Bason 2018). Esta innovación se caracteriza por ser abierta, deliberativa, democrática, colaborativa y ciudadana. 
Abierta, en tanto las fronteras se vuelven porosas, y la "caja negra" de las organizaciones pasa de ser un espacio cerrado a un lugar de intercambio donde los actores entran y salen, y los procesos de innovación pueden generarse en distintos puntos, no solo al interior de la organización sino también, y principalmente, en las intersecciones (Chesbrough 2003).

Deliberativa, ya que se basa en la escucha y el diálogo, lo que permite ampliar los marcos del debate, equilibrar posiciones y fomentar la creatividad y el aprendizaje (Brugué 2014).

Democrática, al reducir la barrera de acceso a la información, incorporando a personas con diversos niveles de conocimientos y distintos bagajes y puntos de vista, ampliando con ello las posibilidades de una "reforma democrática genuina" (Girouard y Sirianni 2014, BergvallKareborn et al. 2009, von Hippel 2005).

Colaborativa, en la medida en la que reúne y conecta diferentes capacidades en torno a objetivos comunes, lo que permite innovar a partir de la combinación de conocimientos ya existentes, trascender sus fronteras y alimentar una dinámica acumulativa que abra la puerta a nuevas innovaciones (Mulgan 2014).

Ciudadana, puesto que, por un lado, parte de una noción de innovación entendida como capacidad universal humana y, por el otro, incorpora durante todo el proceso a la ciudadanía a participar, en especial a los afectados (Freire 2017). En ese sentido, no se trata tanto de encontrar buenas soluciones para la gente, sino de construirlas con la gente misma (Carstensen y Bason 2012).

Experiencias que promuevan la innovación así entendida aumentan las interacciones entre diferentes actores $y$, a partir de ello, incrementan las probabilidades de generar procesos de inteligencia colectiva y valor compartido (Bernstein y Turban 2018, Porter y Kramer 2011), lo que va a condicionar la capacidad de las nuevas instituciones para experimentar, fortalecer redes y prototipar soluciones sostenibles (Smith 2018, Binder y Brandt 2008).

Las preguntas que se abren ahora son: dónde y cómo poner en práctica este tipo de innovación. En relación al dónde, los laboratorios ciudadanos y de gobierno pueden ser un espacio para el encuentro entre actores y el intercambio de información, donde combinar la lógica más desestructurada y espontánea de la innovación con ciertas pautas y regularidades propias de los procesos de institucionalización (Braybrooke y Smith 2018). Se 
conforman como espacios flexibles y de naturaleza híbrida, claves para el desarrollo de la experimentación. Dos de las experiencias que se analizan en este artículo se centran en estos espacios como contexto de innovaciones democráticas.

Sin embargo, promoverel encuentrode actores noconduce necesariamente al diálogo y, menos aún, a prácticas cooperativas. El diseño, seguimiento y evaluación de los programas es clave para afrontar las dificultades y complejidades del diálogo. Escobar (2011) ha identificado algunas barreras específicas en la comunicación, como los distintos lenguajes que se utilizan (más técnicos, más coloquiales), la aparición de voces predominantes o el planteamiento de problemáticas en términos simplistas o de polarización. Para superar estos obstáculos, el diálogo requiere de estímulo por parte de las propias instituciones, así como un ejercicio continuado, a modo de entrenamiento. La función de las instituciones pasa, por tanto, por alentar la escucha y coordinar esfuerzos mediante la identificación de temas prioritarios, recursos disponibles y posibles vías de actuación, haciendo del diálogo una práctica de adaptación cultural que permita a actores muy dispares aproximar posiciones con compromisos estratégicos en torno a objetivos comunes (Smith 2010, Levine, Fung y Gastil 2005).

Conseguido ese diálogo, las actitudes negativas se reducen y se vuelve más probable la cooperación, de forma que: 1) los ciudadanos se sienten involucrados en la toma de decisiones y parte del proceso de las políticas; 2) los decisores y funcionarios públicos cuentan con más información y acceden a espacios ciudadanos a los que de otra forma les sería difícil llegar; 3) se aprovecha mejor las capacidades de cada uno; y 4) todos perciben los beneficios: para los ciudadanos, una administración que responde; para los decisores y funcionarios, una respuesta más eficaz y que cuenta con mayor legitimidad (Berkowitz y Gagnon 2017, Sjoberg, Mellon y Peixoto 2017, Yang y Callahan 2007, Cooper, Bryery y Week 2006, Huxham 2003).

Se trata de una práctica progresiva, de aproximación entre distintos actores que van venciendo recelos y consiguen cooperar desarrollando en el largo plazo confianzas mutuas. Si entendemos la confianza como una ecología de instituciones, actores e interacciones, son las instituciones las que tienen que dar el primer paso para crear confianza: si no se vuelven confiables, será difícil que el resto de actores confíe realmente en ellas y, al mismo tiempo, se vuelvan dignos de confianza. Nuevas instituciones y mecanismos de participación generan confianza y, a su vez, esta confianza refuerza vínculos cívicos y una cultura institucional que consolida dichos procesos (Güemes y Resina 2018). 
A partir de esta reflexión, en el siguiente apartado se abordan tres estrategias institucionales del ámbito local y autonómico en España, que tienen como fin reconectar con la ciudadanía, buscar mayor legitimidad y lograr que la innovación democrática sea una práctica habitual.

\section{EXPERIMENTANDO SOLUCIONES: LOS CASOS DE ARAGÓN, VALENCIA Y MADRID}

Las experiencias que presentamos en este apartado tienen en común que: 1) suponen un intento de democratización de la administración pública; 2) cuentan con un diseńo institucional experimental; 3) promueven nuevas prácticas de innovación; y 4) estimulan relaciones de confianza. Las dos primeras estrategias corresponden a niveles de gobierno autonómico (Aragón y Valencia), mientras que la tercera, al municipal (Madrid). En cuanto a su objetivo, la de Aragón se orienta a fomentar la participación, la de Valencia se centra en la transparencia y la de Madrid en la colaboración

El esquema de análisis que utilizaremos para presentarlas empieza describiendo el punto de partida en el que surge la innovación, sigue con el desarrollo del diseño institucional y, por último, identifica cuáles son los principales aprendizajes, tanto positivos como limitaciones, que deja su implementación.

El punto de partida, primera dimensión, presenta la problemática a la que se quiere responder, el objetivo que se establece, qué actor impulsa el cambio y el contexto inicial del que se parte. Las tres experiencias se inician en un contexto de cambio de gobierno, con el ascenso al poder de coaliciones de centro izquierda tras las elecciones autonómicas y municipales de 2015 en España. Este escenario supone, por un lado, la introducción de nuevas propuestas que buscan ampliar, modificar o profundizar las rutas democráticas y, por el otro, la exigencia de adaptar tales propuestas al entorno institucional previo, así como la necesidad de aprovechar y reutilizar recursos existentes.

El desarrollo de diseño institucional, segunda dimensión de análisis, describe la innovación que se introduce, la meta específica que se persigue y la estrategia planteada. Las tres experiencias se impulsan en un marco en el que el gobierno abierto se instaura como paradigma en la forma de gobernar, y responden a estrategias transversales, que además de incluir varias temáticas, implican a una diversidad de actores institucionales y sociales.

Los principales aprendizajes, tercera dimensión, indican los aspectos positivos y las dificultades surgidas. Al tratarse de innovaciones 
institucionales que tienen todavía un ciclo de vida corto, la valoración ha de entenderse más como un balance preliminar que como una evaluación definitiva. A juzgar por las valoraciones de los responsables de las tres experiencias, el camino recorrido se entremezcla con las expectativas, aunque permite identificar unas primeras enseñanzas. De forma general, en los tres casos se subraya, por un lado, la dificultad de acompasar tiempos políticos y administrativos y de sincronizar intereses, la escasez de recursos con la que se cuenta y la rigidez de las estructuras burocráticas y, por el otro, la importancia que tiene la experimentación de nuevas formas y dispositivos institucionales y la creación de unas estructuras que permitan la supervivencia de estas innovaciones más allá de los cambios de ciclo político.

\section{Gráfico 1: Análisis de las experiencias}
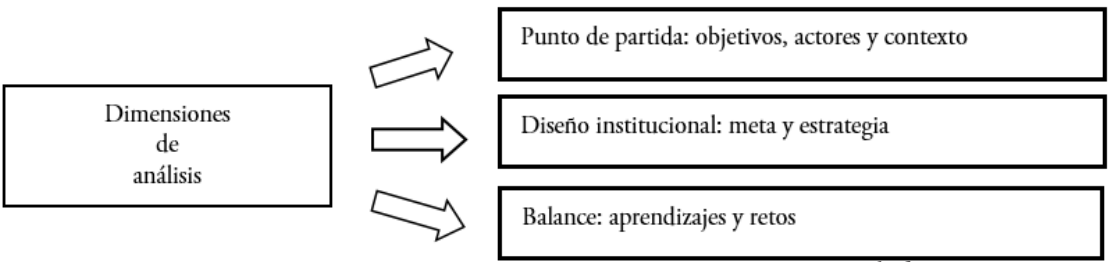

Fuente: Elaboración propia.

La metodología seguida para los dos primeros casos, Aragón y Valencia, ha consistido en entrevistas con las Direcciones Generales de Transparencia y Participación de ambos gobiernos autonómicos, así como en el análisis documental de la información disponible en sus respectivos portales web ${ }^{1}$. Para el tercer caso, Madrid, se retoma la información de tipo cualitativa y primaria (observación participante, entrevistas y encuestas) recolectada por los autores durante la celebración del programa Madrid Escucha (Güemes, Resina y Cruz 2017).

1 Para el caso de Valencia, las entrevistas y conversaciones se entablan con Aitana Mas (19/12/18), Directora de Transparencia y Participación; para el de Aragón con Raúl Olivan, Director de Transparencia y Participación, y Beatriz Palacios (26/12/18), miembro del equipo. El portal web de Aragón es: http://aragonparticipa.aragon. es/ el de Valencia: http:/www.transparencia.gva.es La información analizada en estos portales web se complementó con la información expuesta por los propios responsables en el Güemes, Resina y Cruz Rubio (2018). 


\section{Laboratorio de Aragón [Gobierno] Abierto (LAAAB) Punto de partida}

Durante los últimos años. Aragón ha acumulado un amplio bagaje en participación ciudadana. Ello se debe en buena medida a la aprobación en 2010 del programa Aragón Participa ${ }^{2}$, destinado a promover una mayor implicación de la ciudadanía en la elaboración de políticas públicas a través de un proceso estructurado en tres fases: informativa, debate y retorno.

Desde entonces, distintos han sido los planes y propuestas normativas locales y autonómicas que se han aprobado mediante este procedimiento, que "obligó" tanto al Gobierno de Aragón como a las entidades locales a difundir información sobre los proyectos, escuchar a la ciudadanía, debatir con ella su contenido y dar cuenta de en qué medida se incorporaban sus propuestas en el borrador final.

Junto a esta experiencia, en marzo de 2015, se aprobó la Ley 8/2015, de 25 de marzo, de Transparencia de la Actividad Pública y Participación Ciudadana de Aragón ${ }^{3}$, que sentó las bases para el Gobierno Abierto en Aragón, regulando cuestiones como el acceso a la información pública, la transparencia de la administración y el alcance de la participación ciudadana. Impulsado por la Dirección General de Participación, Transparencia, Cooperación y Voluntariado, de la Consejería de Ciudadanía y Derechos Sociales, Gobierno de Aragón, y en virtud de este nuevo marco normativo, se crean espacios de experimentación, se profundizan los procesos participativos, se mejora su coordinación y se fomentar y concreta el involucramiento de la ciudadanía, al potenciar espacios de confluencia y explorar nuevos modelos de acción pública.

\section{Diseño institucional}

La principal innovación institucional es la creación del Laboratorio de Aragón [Gobierno] Abierto (LAAAB). Su diseño se asemeja a otros laboratorios de gobierno puestos en marcha, como el GovLab en Estados Unidos o el MindLab en Dinamarca, y tiene el propósito de canalizar la participación ciudadana y crear un espacio de experimentación de políticas

2 El programa se encuentra disponible en línea en http://aragonparticipa.aragon.es/ [10-01-2019].

3 Texto de la ley disponible en línea en http://aragonparticipa.aragon.es/sites/default/ files/ley_8_2015_de_transparencia_y_participacion_ciudadana_de_aragon.pdf [1001-2019]. 
que parte de la experiencia de los propios usuarios/afectados, a través de un diseño abierto, con la celebración de talleres, la participación de mediadores y la creación de una plataforma digital de seguimiento y evaluación.

El propósito principal del LAAAB es generar sinergias y aprovechar los recursos y conocimientos disponibles para mejorar políticas e impulsar la innovación pública. Con este fin, combina distintas estrategias presenciales y digitales, de manera que se facilite la coordinación y se eviten duplicidades. Todo el proceso tiene además un carácter abierto, dando cabida a ciudadanía y actores públicos y privados. Su estrategia se compone de cinco grandes ejes de actuación:

Una metodología basada en design thinking (pensamiento de diseño) estructurada en cinco fases (empatía, definición, ideación, prototipado, testeo) con carácter abierto y colaborativo, que incorpora un menú de técnicas de debate, un servicio de mediadores que operan como nexos, un proceso de registro de las actividades y una capa online de apoyo y seguimiento.

- Un espacio físico que funciona como laboratorio ciudadano, con una organización que favorezca el encuentro entre personas y el diálogo, donde se genere un clima "cálido" en el que tanto la ciudadanía como el resto de actores se encuentren cómodos.

- Una red social que integre a los usuarios de los portales de participación, transparencia y acción voluntaria, de forma que el portal web Aragón Participa se parezca más a una gran red social que incluya a todos los actores vinculados a procesos de participación. De este modo, quienes deseen puedan tener un perfil propio con sus datos de contacto y tener acceso a los procesos participativos que se encuentren en marcha, así como a ver sus contribuciones y la documentación generada y hacer seguimiento de políticas.

- Una comunidad de innovación abierta, que genere un espacio de pensamiento, a modo de thinknet (como think tank en red), y registro de experiencias a través de artículos y material audiovisual e hipermedia recogido en un blog, que congregue a expertos y participantes con distintos temas vinculados a innovación abierta, inteligencia colectiva y procesos participativos.

- Un ecosistema de proyectos, que interconecte distintas actividades participativas, como las actividades de formación (Escuela de Participación, con la Universidad de Zaragoza), los encuentros de innovación ciudadana (Campus LAAAB, en colaboración Medialab-Prado y la Secretaría General Iberoamericana), los procesos deliberativos vecinales (G1000. Cumbre Ciudadana), el mapeo de iniciativas de innovación (CIVICS) y las consultas y 
debates ciudadanos a nivel local (Plataforma de participación para Entidades Locales).

\section{Aprendizajes}

Gracias a su naturaleza transversal, el LAAAB ocupa una posición privilegiada e incorpora una nueva capa de diseńo abierto al proceso de elaboración de políticas públicas. El principal reto de sus primeros meses de funcionamiento ha sido introducir en la administración pública una forma de trabajar que tiene la experimentación como leitmotiv. Acorde esto, sus responsables señalan que el éxito de la iniciativa no va a depender tanto de conseguir logros inmediatos, sino de tener la posibilidad de equivocarse:

Pretendemos que el éxito de la implementación del modelo del LAAAB venga dado por la experimentación. No significa que todo lo experimentado tenga éxito; al contrario, la experimentación implica poder equivocarse y fracasar. Sin embargo, un laboratorio para experimentar en políticas públicas generará un nuevo modelo de hacer (Oliván, Tráid y PalaciosLlorente 2018: 93).

Varios han sido los avances del Laboratorio, y en distintas direcciones. Bajo su marco, se ha puesto en marcha una comunidad de innovación abierta que cuenta con 70 personas expertas en temas de participación y ha comenzado a funcionar un blog que recoge sus aportaciones; se ha empezado a trabajar en un proyecto piloto para rediseñar de forma participativa y a través de la experiencia de usuario una oficina del Instituto Aragonés de Empleo (INAEM); y también se ha acompañado y apoyado a las Entidades Locales a través de la herramienta Nexo-LAAAB, una plataforma de participación online para mejorar la calidad de la deliberación y la representación de los procesos locales.

Este proceso ha ido también acompañado de dificultades, sobre todo en la conciliación entre una nueva forma de trabajo experimental y los procedimientos y tiempos político-administrativos. Por un lado, están los plazos reducidos que fijan las instituciones para celebrar procesos participativos, lo que dificulta su profundización y una mayor deliberación. Por el otro, los tiempos políticos, ya que en el último periodo de legislatura no se inician planes o proyectos normativos autonómicos acompañados de un proceso deliberativo de participación ciudadana. Este hecho ha impedido que el LAAAB pudiera tener mayor actividad al respecto.

También han existido otras dificultades, más sustantivas. Entre ellas, las reticencias políticas e institucionales para abrir temas a la participación 
ciudadana. Por lo general, desde ámbitos tanto administrativos como políticos se considera que son temáticas que requieren de conocimiento experto y que la ciudadanía no está capacitada. Otro aspecto es la dificultad para adaptar los recursos técnico-tecnológicos y humanos existentes a nuevas tareas, que suponen además un cambio en la forma tradicional de operar de la administración pública. Por último, y no menos importante, la gestión de expectativas y las frustraciones ciudadanas que se generan en los distintos procesos participativos, puesto que muchos de los participantes acuden con altas expectativas y si no se cumplen, terminan frustrados, lo que provoca a medio plazo un escepticismo hacia los procesos participativos.

\section{El sistema de transparencia de la Generalitat Valenciana Punto de partida}

La cantidad y la magnitud de casos de corrupción que salieron a la luz en Valencia durante la VII y VIII Legislatura (2007-2015) llevó a la dimisión en 2011 del entonces Presidente de la Generalitat Francisco Camps, al ser investigado por la justicia espańola por su posible relación en varios de los casos. En paralelo, varios diputados de su formación, el Partido Popular, renunciaron a su escaño, produciendo una situación insólita, ya que durante la VIII Legislatura el partido llegó a tener más de una decena de imputados por delitos de estafa, malversación y soborno en las Cortes Valencianas ${ }^{4}$. Esta situación llevó a que, tras las elecciones de mayo de 2015, el nuevo gobierno, a través de los denominados Acuerdos del Botànic, priorizara la transparencia y la lucha contra la corrupción como uno de sus principales ejes programáticos ${ }^{5}$.

Para ello, el nuevo gobierno: 1) creó la Dirección General de Transparencia y Participación, dentro de la nueva Conselleria de Transparencia, Responsabilidad Social, Participación y Cooperación; 2) desarrolló el reglamento de aplicación de la Ley 2/2015, de 2 de abril, de Transparencia, Buen Gobierno y Participación Ciudadana de la Comunitat

4 Véase "Los 17 casos de corrupción que han hundido al PP en Valencia” (El Español, 22/05/2018). Disponible en línea en https://www.elespanol.com/espana/20180522/ casos-corrupcion-hundido-pp-valencia/309219868_0.html y "Quedan seis diputados imputados del PP en las Cortes Valencianas” (El Mundo, 21/03/2014). Disponible en línea en https:/www.elmundo.es/comunidad-valenciana/2014/03/21/532c229d226 01dda178b456f.html [10-01-2019].

5 El texto del "Acuerdo del Botànic" disponible en línea en https://www.larazon.es/ documents/10165/0/video_content_3210271_20150611180323.pdf [10-01-2019]. 
Valenciana, aprobada justo antes de las elecciones de $2015^{6}$; y 3) puso en marcha mecanismos novedosos contra la corrupción, para fomentar la cultura de la transparencia dentro de la administración pública y la actividad gubernamental.

\section{Diseño institucional}

Entre los nuevos mecanismos, la Generalitat diseñó una tecnología anticorrupción basada en herramientas digitales de carácter preventivo y de anticipación sobre malas prácticas administrativas en la gestión pública. A través de esta tecnología se permite el monitoreo de datos e información pública, la identificación de riesgos y la instauración de un sistema de alertas en tiempo real de la tramitación de expedientes, de forma que se mejora la detección de posibles casos e indicios de prácticas corruptas, mediante procedimientos simples y directos. Con esta herramienta, el propósito del gobierno, más allá de la detección de posibles irregularidades, es instaurar un sistema integral con efectos a medio y largo plazo, de forma que se fomente una cultura de transparencia.

De esta forma, la puesta en marcha de esta herramienta digital ha de entenderse inserta dentro de una estrategia más general de transparencia gubernamental y administrativa. Por ejemplo, la formación de los empleados públicos en materias de gobierno abierto ha sido clave para crear una nueva cultura administrativa. Para ello, se diseñaron cursos presenciales y online, y se crearon unidades de transparencia formada por 2-3 interlocutores en cada conselleria. También la aprobación de un código de conducta de buen gobierno para altos cargos ha sido otro de los puntales de esta estrategia, con el fin de que se autolimiten (no pueden tener cuentas en paraísos fiscales ni pueden disponer de una tarjeta de crédito institucional, entre otras cuestiones) y transparenten su actuación en el ejercicio público (desde su agenda hasta los regalos institucionales que reciben $)^{7}$.

Otras actividades han formado también parte de esta estrategia. Por un lado, aquellas orientadas a la sociedad, con programas como Palaus Transparents, por el que la ciudadanía puede visitar las instituciones de

6 Ley disponible en línea en https://boe.es/buscar/pdf/2015/BOE-A-2015-4547consolidado.pdf [10-01-2019].

7 El texto del Código de Buen Gobierno de la Generalitat está disponible en línea en http://www.gvaoberta.gva.es/es/buen-gobierno-en-la-generalitat;jsessionid=944592 A421E366FF86EA9716014644E3 [10-01-2019]. 
gobierno para conocer su actividad, o Diàlegs d'Estiu, encuentros abiertos en los que miembros del gobierno valenciano rinden cuentas ante los ciudadanos. Por el otro, las más procedimentales, como la creación de una Oficina de Control de Conflictos de Intereses y la aprobación de un marco normativo que fortalezca jurídicamente la transparencia y la lucha contra la corrupción ${ }^{8}$.

Estas acciones se acompañaros también con otras herramientas digitales. En ese sentido, se lanzó un portal transparencia y de datos abiertos que permite solicitar información pública y monitorear la actividad administrativa por temáticas. Se creó un Sistema de Alertas Rápidas (SALER) para prevenir la corrupción, que consiste en una aplicación que cruza bases de datos (sobre contratos y licitaciones, subvenciones, actividad de altos cargos) con algoritmos introducidos previamente acorde a conceptos y premisas legales, de forma que el sistema identifica posibles irregularidades o indicios de malas prácticas. Y, por último, se instauró un buzón de denuncias anónimas que permite a cualquier persona denunciar un abuso o hecho de corrupción de forma anónima, gracias a un sistema de encriptación para que no se conozca la IP del denunciante?

\section{Aprendizajes}

Desde la Dirección General de Transparencia y Participación, se valora positivamente los primeros ańos de funcionamiento de los nuevos mecanismos, ya que tanto ciudadanía como responsables públicos se están "acostumbrando" a un nivel alto de transparencia. El sistema puesto en marcha está impregnando de una nueva cultura a las instituciones, que incorporan aspectos cruciales como publicar agendas de trabajo de los altos cargos, su patrimonio, sus gastos o los regalos que reciben. Esta cultura va acompañada además de una estructura jurídica y orgánica que permite concretarla y que se mantenga con independencia de quien gobierne. En ese sentido, la introducción de nuevos mecanismos que detecten la

8 El Palaus Transparents cuenta con 27 edificios oficiales. Disponible en línea en http:// www.transparencia.gva.es/es/palaus-transparents Sobre programa y las actividades Diàlegs d'Estiu veasé http://www.forumestiu.gva.es/es/dialegs-d-estiu [10-01-2019]. Dentro del marco normativo, el reglamento de desarrollo de la Ley de Transparencia, aprueba la Ley 8/2016 de Incompatibilidades y Conflictos de Intereses de personas con cargos públicos no electos, y la Ley 25/2018, de 10 de diciembre, de la Generalitat, reguladora de la actividad de los grupos de interés de la Comunitat Valenciana.

9 En relación al portal transparencia y de datos abiertos véase http://www.gvaoberta. gva.es/ y en lo que atañe al buzón de denuncias anónimas véase: https://www. antifraucv.es/denuncia/bustia-de-denuncies/) Disponible en línea [10-01-2019]. 
corrupción junto a cursos de formación ha supuesto un aprendizaje para los empleados públicos, así como un cambio de percepción de las prácticas de la administración pública.

En síntesis, se logra introducir como filosofía de funcionamiento administrativo el gobierno abierto, entendido como eje vertebrador de las políticas, obligando a todas las consellerias a abrir sus datos, transparentar su actividad y generar espacios de participación ciudadana. Se sientan también las bases de una cultura de la transparencia, en la que tanto empleados públicos, como políticos y ciudadanía se familiarizan con prácticas transparentes y se reconoce como un derecho ciudadano y un deber de la administración. Además, se logra una muy buena recepción por parte de los funcionarios, que muestran interés por formarse y por contribuir a mejorar las prácticas administrativas.

Las principales dificultades residen en la falta de recursos públicos para poder implementar el sistema, sobre todo a la escasez de personal. Muchos funcionarios se están jubilando y sus puestos quedan vacantes, sin cubrir, al no convocarse oposiciones para su reemplazo.

\section{MADRID EsCUCHA \\ Punto de partida}

Para entender cómo surge y a qué necesidades responde Madrid Escucha como programa de innovación del Ayuntamiento de Madrid, es necesario contextualizar primero la experiencia en base a dos procesos: el marco institucional en el que se inserta, Medialab-Prado, y la llegada en 2015 de un nuevo equipo de gobierno municipal, que tiene la participación como eje de acción.

Por un lado, Medialab-Prado es un laboratorio ciudadano fundado en 2007 que funciona como espacio de experimentación y aprendizaje colaborativo para la producción, investigación y difusión de proyectos en gobierno abierto, orientado al trabajo en red, el procomún y la realización de prototipos. Desde su fundación, se han celebrado diversos laboratorios cívicos, creando una metodología propia basada en la colaboración entre personas con distintos saberes y la combinación de la tecnología digital con otros tipos de conocimientos.

Por otro lado, está la llegada de un nuevo gobierno que tiene como objetivo prioritario impulsar la participación ciudadana. Según Font (2017), puede caracterizarse como la propuesta más rompedora del ámbito municipal en España, al apostar por distintas modalidades participativas. 
Entre esas iniciativas, por su alcance, destaca el programa Decide Madrid, con referendos vinculantes como los celebrados para reformar espacios urbanos de gran calado como Plaza España o Gran Vía (Sánchez Medero y Pastor Albaladejo 2018) o la elaboración de presupuestos participativos, así como otros programas como Experimenta Distrito, que tiene como objetivo crear laboratorios ciudadanos "localizados", en los distintos distritos de la ciudad, con el fin de crear espacios de encuentro entre vecinos para que puedan presentar proyectos y participar en el desarrollo de su barrio ${ }^{10}$.

\section{Desarrollo institucional}

Es dentro del contexto descrito que se lanza Madrid Escucha, como un programa que tiene por objetivo crear espacios de encuentro y diálogo entre ciudadanía y empleados públicos para promover la colaboración en el diseño de prototipos y la búsqueda de soluciones que mejoren la vida de la ciudad. El propósito, en ese sentido, es claro: mejorar la respuesta a las demandas sociales por parte de la administración pública, involucrando a la ciudadanía en el diseño de políticas, de forma que se combine el conocimiento técnico de los empleados públicos con el punto de vista y los saberes ciudadanos.

El programa tuvo dos ediciones y en ambas arrancó con una convocatoria pública para que tanto ciudadanos como empleados públicos presentaran iniciativas con propuestas de mejora de la ciudad en general (primera edición 2017) y centradas en la movilidad urbana (segunda edición 2019). Una vez seleccionadas las iniciativas que formaron parte del programa, se abrió una nueva convocatoria para aquellas personas que quisieran participar como colaboradores en una de ellas ${ }^{11}$.

La fase presencial de los programas consta de ocho sesiones de trabajo y se desarrolla siguiendo la metodología de trabajo desarrollada por Medialab-Prado. Cada grupo se compone por la persona o grupo de personas que propone la iniciativa (promotor) más los colaboradores que voluntariamente se apuntan, y se reúne en mesas de trabajos que cuentan

10 Más información sobre las actividades de Medialab-Prado disponible en línea en https://www.medialab-prado.es/, sobre Decide Madrid disponible en https:// decide.madrid.es/, sobre Experimenta Distrito disponible en línea en https://www. experimentadistrito.net/ [10-01-2019].

11 Para más información sobre las propuestas de las dos convocatorias, véase: https:// www.medialab-prado.es/programas/madrid-escucha [10-01-2019]. 
con el apoyo de mentores, cuya función es orientar, mediar y dinamizar el trabajo de los grupos, y funcionarios "especialistas" del Ayuntamiento de Madrid que acuden en momentos puntuales del laboratorio para asesorar a los grupos sobre cuestiones de carácter técnico y jurídico.

El programa se diseñó para: 1) reunir en un mismo espacio a empleados públicos con ciudadanos, y ponerles a trabajar juntos en los grupos, de modo que compartieran objetivos y se incitara a la cooperación; 2) fomentar la escucha por parte de ambos lados, como paso previo para que se produjese el diálogo; para ello, se trabajó la empatía, se incluyeron en un mismo plano los diversos puntos de vista y se favoreció el intercambio de conocimientos y saberes; y 3 ) incorporar una perspectiva práctica, orientada al co-diseño de un prototipo experimental con el que dar respuesta a la problemática planteada.

\section{Aprendizajes}

El principal logro del programa es que incorpora a la administración pública una nueva dinámica de trabajo, basada en la metodología de laboratorio de innovación, de forma quelas necesidades de los ciudadanos se incluyen desde el inicio en el diseño de políticas, y ambos lados (funcionarios y ciudadanía) se enriquecen con una visión más compleja de las problemáticas. De este modo, se avanza hacia un modelo de administración pública deliberativa, que escucha y dialoga, y los empleados públicos comienzan a asumir un rol de mediación, favoreciendo además el intercambio de información y estableciendo las bases para la confianza.

La fortaleza más notable del programa es que consigue poner a trabajar juntos en un mismo espacio a funcionarios y ciudadanos, ya sea de forma experimental, en soluciones que mejoren la calidad de vida en la ciudad. Junto a ello, permitió generar redes de contacto, de forma que más allá del laboratorio, ciudadanos y funcionarios se conozcan y puedan iniciar una relación de colaboración. Además, los ciudadanos comprenden mejor la estructura de la administración y pueden identificar a qué organismos y empleados públicos tienen que dirigirse para avanzar en un tema en concreto; de la misma manera, desde la administración pública se identifican a actores clave en las distintas áreas de actuación municipal.

Por el lado de las limitaciones, la primera barrera es la dificultad de implicar a más funcionarios en laboratorios de este tipo. En el caso de Madrid Escucha, la participación de funcionarios fue bastante más baja que la de ciudadanos, y su compromiso limitado, ya que quienes acudieron lo hicieron sobre todo porque conocían a los organizadores del programa. 
Otra dificultad es conseguir que participen funcionarios con capacidad de decisión y margen presupuestario, puesto que los participantes en el laboratorio tuvieron un perfil técnico, sin demasiado margen de acción.

Por el lado de la ciudadanía, el principal problema es el sesgo participativo. En su mayoría, los participantes que acudieron al laboratorio tenían experiencia en procesos participativos, formaban parte de una asociación o habían tenido ya contactos con la administración pública, además de existir una brecha socio-económica, por la que casi todos los participantes contaban con estudios de educación superior y vivían en zonas centrales de Madrid.

Si se atiende a las interacciones, reducir el escepticismo por ambos lados fue otra dificultad, ya que tanto funcionarios como ciudadanos acudieron con prejuicios sobre el otro, de igual forma en diferentes fases del laboratorio se mantuvieron recelos mutuos. En cuanto al resultado de los talleres, existe un margen interesante de mejora para concretar los prototipos e insertarlos en la línea de acción municipal, ya que en algunos casos las iniciativas eran o demasiado generales o colisionaban con cuestiones ya gestionadas o quedaban reducidas a micro-proyectos. En ese sentido, es importante que haya sincronía entre las propuestas seleccionadas y la agenda del Ayuntamiento, para poder dar continuidad y seguimiento a los proyectos después del laboratorio. 
Tabla 2: Dimensiones de análisis de las experiencias

\begin{tabular}{|c|c|c|c|c|}
\hline \multicolumn{2}{|c|}{ Dimensiones de Análisis } & Aragón & Valencia & Madrid \\
\hline \multirow[t]{4}{*}{$\begin{array}{c}\text { Punto } \\
\text { de Partida }\end{array}$} & Problemática & $\begin{array}{l}\text { Participación } \\
\text { ciudadana }\end{array}$ & $\begin{array}{l}\text { Transparencia y } \\
\text { lucha contra la } \\
\text { corrupción }\end{array}$ & $\begin{array}{l}\text { Colaboración } \\
\text { funcionarios y } \\
\text { ciudadanía }\end{array}$ \\
\hline & Objetivo & $\begin{array}{l}\text { Profundizar y } \\
\text { abrir procesos } \\
\text { participativos }\end{array}$ & $\begin{array}{c}\text { Crear } \\
\text { mecanismos de } \\
\text { control }\end{array}$ & $\begin{array}{l}\text { Generar espacios } \\
\text { de encuentro }\end{array}$ \\
\hline & Actor & $\begin{array}{c}\text { Dirección General } \\
\text { de Participación, } \\
\text { Transparencia, } \\
\text { Cooperación y } \\
\text { Voluntariado }\end{array}$ & $\begin{array}{c}\text { Dirección } \\
\text { General de } \\
\text { Transparencia y } \\
\text { Participación }\end{array}$ & $\begin{array}{l}\text { Medialab-Prado } \\
\text { (Ayuntamiento de } \\
\text { Madrid) }\end{array}$ \\
\hline & Contexto & $\begin{array}{c}\text { Bagaje en procesos } \\
\text { participativos }\end{array}$ & $\begin{array}{l}\text { Casos de } \\
\text { corrupción } \\
\text { abiertos }\end{array}$ & $\begin{array}{l}\text { Programa de } \\
\text { participación } \\
\text { directa }\end{array}$ \\
\hline \multirow[t]{3}{*}{ Experimentación } & Innovación & $\begin{array}{c}\text { LAAAB: } \\
\text { Laboratorio de } \\
\text { Gobierno [Aragón] } \\
\text { Abierto }\end{array}$ & $\begin{array}{c}\text { Sistema de } \\
\text { Alertas Rápidas } \\
\text { (SALER) y } \\
\text { buzón de } \\
\text { denuncias }\end{array}$ & $\begin{array}{l}\text { Madrid Escucha: } \\
\text { Laboratorio } \\
\text { de Innovación } \\
\text { Ciudadana }\end{array}$ \\
\hline & Meta & $\begin{array}{l}\text { Aprovechar } \\
\text { conocimientos } \\
\text { ciudadanos }\end{array}$ & $\begin{array}{l}\text { Prevención } \\
\text { rápida de } \\
\text { prácticas } \\
\text { corruptas }\end{array}$ & $\begin{array}{c}\text { Mejorar escucha y } \\
\text { diálogo }\end{array}$ \\
\hline & Estrategia & $\begin{array}{c}\text { Interconectar } \\
\text { actores y fomentar } \\
\text { un ecosistema } \\
\text { de innovación, } \\
\text { que combine lo } \\
\text { presencial y lo } \\
\text { digital }\end{array}$ & $\begin{array}{c}\text { Integrar } \\
\text { herramientas } \\
\text { digitales a la } \\
\text { formación de } \\
\text { funcionarios y la } \\
\text { creación de un } \\
\text { marco jurídico }\end{array}$ & $\begin{array}{l}\text { Poner a trabajar } \\
\text { de forma conjunta } \\
\text { a funcionarios y } \\
\text { ciudadanos para } \\
\text { que el co-diseño } \\
\text { de prototipos }\end{array}$ \\
\hline \multirow[t]{3}{*}{ Balance } & $\begin{array}{l}\text { Aprendizaje } \\
\text { Institucional }\end{array}$ & $\begin{array}{c}\text { Experimentación y } \\
\text { diseño abierto }\end{array}$ & $\begin{array}{l}\text { Cultura de la } \\
\text { transparencia }\end{array}$ & $\begin{array}{c}\text { Administración } \\
\text { deliberativa }\end{array}$ \\
\hline & Avances & $\begin{array}{l}\text { Creación de una } \\
\text { comunidad de } \\
\text { innovación }\end{array}$ & $\begin{array}{l}\text { Apoyo y } \\
\text { buena acogida } \\
\text { funcionarios }\end{array}$ & $\begin{array}{l}\text { Generación de } \\
\text { redes de contactos }\end{array}$ \\
\hline & Dificultades & $\begin{array}{l}\text { Tiempos y plazos } \\
\text { limitados }\end{array}$ & $\begin{array}{l}\text { Escasez de } \\
\text { recursos } \\
\text { humanos } \\
\text { Fuente: } I\end{array}$ & $\begin{array}{c}\text { Sesgo participativo } \\
\text { y recelos }\end{array}$ \\
\hline
\end{tabular}

\section{TRES INNOVACIONES PARA EL CAMBIO CULTURAL}

Si se evalúa el grado de institucionalización de las herramientas descritas en términos democráticos y de gobierno abierto, puede señalarse que, para el caso de Aragón, la creación de un laboratorio de gobierno dentro de la propia administración pública autonómica está permitiendo generar un 
espacio novedoso de transformación y puesta en común de conocimientos.

En Valencia, se operacionaliza el cambio desarrollando una plataforma anticorrupción (apuesta técnica) que nace de un pacto político y que se complementa con cursos de sensibilización que, a la vez que introducen prácticas de transparencia (apertura y publicación de datos), identifican acciones correctas (qué es y qué no corrupción), buscando socializar y expandir nuevas pautas de conducta.

En el caso de Madrid, se aprovecha la experiencia y la reputación con la que cuenta un espacio híbrido como Medialab-Prado, y en su seno se desarrolla un programa enfocado a promover el diálogo entre ciudadanía $\mathrm{y}$ funcionarios, al tiempo que revertir estereotipos y fomentar nuevas prácticas de trabajo.

En cuanto al modo en que dichas innovaciones reconfiguran la cultura burocrática y buscan fomentar confianzas mutuas, para Aragón, el planteamiento es crear un ecosistema de innovación que tiene como centro un laboratorio. A partir de él, se busca irradiar y distribuir la innovación hacia toda organización. La apuesta consiste en que este espacio sirva para divulgar conocimiento experto (notas y columnas), experiencias de otras latitudes (buenas prácticas) y estrategias experimentales donde "hacer juntos" (learning by doing, aprender haciendo). Se pretende transformar la organización pública para, a partir de ahí, entablar nuevas relaciones con la ciudadanía y que esta reciba el mensaje y responda de nuevas maneras.

En el caso de Valencia, también el cambio de cultura se piensa de dentro hacia fuera: cambiar la organización para que esto redunde en un cambio de expectativas, actitudes y percepciones de la ciudadanía hacia ella. Aquí la apuesta fuerte viene en la transparencia, en regenerar la institución y demostrar a la ciudadanía que hay nuevas reglas del juego, y las instituciones pueden ser merecedoras de confianza.

En Madrid, la propuesta es diferente. Desde un espacio que se percibe como campo neutral (aunque pertenece al Ayuntamiento), se invita a participar en talleres vivenciales a la ciudadanía y a los funcionarios. La apuesta es generar efecto arrastre y contagio tanto en la administración pública local (que los funcionarios que no participaron quieran hacerlo en futuras ediciones y que quienes participaron trasladen lo aprendido dentro de su institución) como en la ciudadanía, para que descubra en estos nuevos espacios un lugar donde canalizar demandas y tener capacidad decisoria. 


\section{CONCLUSIONES: UN PROCESO QUE REQUIERE DECISIÓN... Y PACIENCIA}

El proceso de institucionalización de la innovación democrática conlleva la cristalización de prácticas, el asentamiento de rutinas y la normalización de patrones de conducta. En ese sentido, las instituciones son conservadoras y se vuelven temerosas al cambio, máxime si este promete una transformación estructural que suponga una nueva cultura organizativa.

Como señala Mulgan (2007), en muchas ocasiones la innovación se inicia casi de forma forzada, desde el exterior, presionada por nuevas demandas sociales o avances tecnológicos. Aunque, como señala Oliván (2018), para que esta innovación sea posible es necesario que alguien abra la puerta desde dentro, un hacker insider capaz de decodificar la institución e iniciar el proceso de apertura. Abierta esa puerta, comienza el camino que da lugar a un círculo virtuoso de participación, que empieza siempre con la incorporación de la ciudadanía, y termina transformando las organizaciones en instituciones híbridas, donde las capacidades burocráticas y de respuesta al mercado se conjugan con el control democrático de base de las administraciones públicas (Evans 2003).

$\mathrm{Al}$ inicio de este artículo planteábamos una serie de preguntas sobre cómo concretar dichas innovaciones, qué formato habrían de tener y si crean o no confianza para, en última instancia, cuestionarnos sobre si realmente merece la pena emprender tal camino. Como hemos visto, la urgencia de las demandas sociales y la necesidad de relegitimar a las instituciones llevan a estas a apostar por un punto de vista pragmático en donde no existe un manual sobre qué forma concreta han de tener estos procesos de apertura y democratización, lo que permite un amplio margen de experimentación, donde el ensayo, el error y la prueba son parte fundamental.

Esto hace que las innovaciones institucionales comiencen con una pequeña ruptura en la organización que puede llevar a un gran cambio, pero que ha de entenderse como un proceso paulatino. A ese respecto, las nuevas tendencias sociales van, poco a poco, permeando a las administraciones que empiezan, no sin recelos, a adoptar nuevas formas de relacionarse y a probar formatos experimentales, ya sea en forma de laboratorio de gobierno, de tecnología anti-corrupción o de espacios de co-creación, como sucedía en los casos presentados.

Estas experimentaciones, al interior de la organización, implican un cambio de pautas y una nueva auto-percepción sobre cuál es la misión y las funciones de la institución y de quienes forman parte de la misma. Ese cambio de chip es el primer paso para, como se señalaba, convertir en 
confiables a las instituciones. Aunque ello no está exento de dificultades. Como se recoge tanto en el debate teórico como en las propias experiencias, las limitaciones siguen ahí y son muchas y variadas: desde el escepticismo de propios y ajenos, hasta las dificultades de sincronizar tiempos políticos, administrativos y ciudadanos, pasando por la sempiterna escasez de recursos económicos y las complejidades de adaptar el personal humano disponible a nuevas funciones que, para mayor dificultad, son experimentales.

Precisamente, esta escasez de recursos supone uno de los grandes baches para invertir en estos procesos. La ausencia de resultados inmediatos y la falta de continuidad pueden conducir a un callejón sin aparente salida. Esperar sin ansiedad es clave para el éxito de innovaciones como las aquí presentadas: revertir la desconfianza ciudadana y convertirla en una relación saludable, en la que se estrechen los vínculos y se entrene el músculo cívico, requiere de tiempo y un trabajo continuado. No es casualidad que tanto la directora de participación de Valencia como el de Aragón, Aitana Mas y Raúl Oliván, coincidan en señalar que su meta última es hacer de estos procesos una realidad irreversible. 


\section{REFERENCIAS}

Accetti, C. I., Mulieri, A. et al. (2016). Debating representative democracy. Contemporary Political Theory, 15 (2), 205-242.

Aguilar Villanueva, L. (2006). Gobernanza y gestión pública. México: Fondo de Cultura Económica.

Bason, Christian (2018). Leading public sector innovation: Co-creating for a better society. Bristol: Policy Press at the University of Bristol.

Bergvall-Kareborn et al. (2009). Living Lab: an open and citizen-centric approach for innovation. International Journal of Innovation and Regional Development, 1 (4), 356-370.

Berkowitz, B., y Gagnon, J. P. (2017). SeeClickFix empowers citizens by connecting them to their local governments. Democratic Theory, 4 (1), 121-124.

Bernstein, E. S. y Turban, S. (2018). The impact of the 'open' workspace on human collaboration. Philosophical Transactions of the Royal Society, 373 (1753), 1-8.

Binder, T. y Brandt, E. (2008). The Design: Lab as platform in participatory design research. CoDesign, 4 (2),115-129.

Braybrooke, K. y Smith, A. (2018). Liberatory technologies for whom? Exploring a new generation of makerspaces defined by institutional encounters. The Journal of Peer Production, 12, 1-12.

Brugué, Q. (2004). Modernizar la administración desde la izquierda: burocracia, nueva gestión pública y administración deliberativa. Revista del CLAD Reforma y Democracia, 29, 27-56.

. (2009). Una administración que habla es una administración que piensa. En Celaya, I. et al. (Coords.), Participación Ciudadana... para una administración deliberativa. Aragón: Gobierno de Aragón.

. (2014). Políticas públicas: entre la deliberación y el ejercicio de autoridad. Revista Cuadernos de Gobierno y Administración Pública, 1 (1), 37-55. 
Cabrero, E. y Arellano, D. (1993). Análisis de innovaciones exitosas en organizaciones públicas. Una propuesta metodológica. Gestión y Política Pública, 2 (1), 59-86.

Carstensen, H. V. y Bason, C. (2012). Powering collaborative policy innovation: Can innovation labs help? The Innovation Journal: The Public Sector Innovation Journal, 17(1), 2-26.

Castoriadis, C. (1996). La democracia como procedimiento y como régimen. Iniciativa Socialista, 38, 50-59.

Chesbrough, H. (2003). Open innovation: The new imperative for creating and profiting from technology. Cambridge: Harvard Business School Press.

Coleman, S., Pzybylska, A. y Sintomer, Y (2015). Deliberation and democracy: innovative processes and institutions. Frankfurt: Peter Lang Editions.

Cooper, T. L., Bryer, T. A. y Meek, J. (2006). Citizen-centered collaborative public management. Public Administration Review, 66 (1), 76-88.

Cornwall, A. (2004). Introduction: New democratic spaces? The politics and dynamics of institutionalised participation. IDS Bulletin, 35 (2), $1-10$.

Della Porta, D. (2013). Can democracy be saved? Participation, deliberation and social movements. Cambridge: Polity Press.

Elster, J. (1998). Deliberation and constitution making. En Elster, J. (Ed.), Deliberative democracy. Cambridge: Cambridge University Press.

Escobar, O. (2011). Public dialogue and deliberation: A communication perspective for public engagement practitioners. Edinburgh: Beacons for Public Engagement.

. (2017). Pluralism and democratic participation: what kind of citizen are citizens invited to be? Contemporary pragmatism, 14 (4), 416-438.

Evans, P. (2003). El hibridismo como estrategia administrativa: combinando la capacidad burocrática con las señales de mercado y la democracia deliberativa. Revista del CLAD Reforma y Democracia, 25, 1-15. 
Font, J., Wojcieszak, M. y Navarro, C. J. (2015). Participation, representation and expertise: Citizen preferences for political decisionmaking processes. Political Studies, 63 (1), 153-172.

. (2017). Nuevos mecanismos participativos: un concepto, distintas realidades. Recerca, Revista de Pensament i Anàlisi, 21, 131135 .

Freire, J. (2017). Innovación ciudadana vs. innovación social. Disponible en http://juanfreire.com/innovacion-social-vs-innovacion-ciudadana/ [10-01-2019].

Fung, A. y Wright, E. O. (2001). Deepening democracy: innovations in empowered participatory governance. Politics \& Society, 29(1), 5-41.

Girouard, J. y Sirianni, C. (Eds.) (2014). Varieties of civic innovation: Deliberative, collaborative, network, and narrative approaches. Nashville: Vanderbilt University Press.

Güemes, C. y Resina, J. (2018). Participación ciudadana y confianza, un ejercicio de alquimia democrática. En Güemes, C., Resina, J. y CruzRubio, C. (Eds.), Participación ciudadana: Experiencias inspiradoras en España. Madrid: CEPC-GIGAPP.

Güemes, C, Resina, J., y Cruz, C. (2017) Informe preliminar de la investigación. Disponible en línea en http://madridescucha.medialabprado.es/informe-madrid-escucha-2017 [10-01-2019].

Güemes, C., Resina, J. y Cruz-Rubio, C. (Eds.) (2018). Participación ciudadana: Experiencias inspiradoras en España. Madrid: CEPCGIGAPP.

Huxham, C. (2003). Theorising collaboration practice. Public Management Review, 5(3), 401-424.

Jessop, B. (1996). Post-Fordism and the state. En Greve, B. (Ed.), Comparative Welfare Systems. Basingstoke: Palgrave Macmillan.

Levine, P., Fung, A. y Gastil, J. (2005). Future directions for public deliberation. Journal of Public Deliberation, 1(1), 1-13.

Mayntz, R. (2001). El Estado y la sociedad civil en la gobernanza moderna. Revista CLAD Reforma y Democracia, 21, 1-8. 
Mulgan, G. (2007). Social innovation: What it is, why it matters and how it can be accelerated. Oxford: Skoll Centre for Social Entrepreneurship.

Mulgan, G. (2014). The radical's dilemma: an overview of the practice and prospects of Social and Public Labs. Sin ciudad: Nesta.

Oliván, R. (2018). Introducción. Abrir instituciones desde dentro [Hacking Inside Black Book]. En Equipo LAAAB, Traid, E., Barriga, S., Palacios, B., Jesús Isarre, J. y Oliván, R-(Coords.), Abrir instituciones desde dentro, Hacking Inside Black Book. Disponible en línea en http:// www.laaab.es/hackinginside/ [10-01-2019].

Oliván, R., Traíd, E. y Palacios-Llorente, B. (2018). LAAAB. Laboratorio de Aragón [Gobierno] Abierto. En Güemes, C., Resina, J. y CruzRubio, C. (Eds.),. Participación ciudadana: Experiencias inspiradoras en España. Madrid: CEPC-GIGAPP.

Pollitt, C., Bouckaert, G. y Löffler, E. (2007). Making quality sustainable: Co-design, co-decide, co-produce, co-evaluate. Helsinki: Ministry of Finance.

Porter, M. y Kramer, M. (2011). La creación de valor compartido. Harvard Business Review América Latina, reimpresión R1101C-E.

Putnam, R. D. (2001). Bowling alone: The collapse and revival of American community. Nueva York: Simon and Schuster.

Ramió, C. (2017). La administración pública del futuro (horizonte 2050): Instituciones, politica, mercado y sociedad de la innovación. Madrid: Editorial Tecnos.

Sánchez Medero, G. y Pastor Albaladejo, G. (2018). The quality of participatory processes in the urban redevelopment policy of Madrid city council. Lex Localis. Journal of Local Self-Government, 16 (4), 841 872.

Sirianni, C. (2009). Investing in democracy, engaging citizens in collaborative governance. Washington: Brooking Institutions Press.

Sjoberg, F. M., Mellon, J. y Peixoto, T. (2017). The effect of bureaucratic responsiveness on citizen participation. Public Administration Review, 77(3), 340-351. 
Smith, A. (2018). Forking the SDGS: How prototypes could transform the new global goals. Disponible en https://steps-centre.org/blog/forkingsdgs-prototypes-transform-new-global-goals/ [10-01-2019]

Smith, N. (2010). The public administrator as collaborative citizen: three conceptions. Public Administration Quarterly, 34 (2), 238-261.

Subirats, J. (2000). Democracia, participación y eficiencia. Foro Internacional, 40 (3), 430-450.

Von Hippel, E. (2005). Democratizing innovation. Cambridge: The MIT Press.

Waylen, G. (2015). Engendering the 'crisis of democracy': Institutions, representation and participation. Government and Opposition, 50(3), 495-520.

Welp, Y. y Ordóńez, V. (2017). La democracia directa a debate: procesos y mecanismos de participación ciudadana. Recerca, Revista de Pensament i Anàlisi, 21, 9-14.

Yang, K. y Callahan, K. (2007). Citizen involvement efforts and bureaucratic responsiveness: Participatory values, stakeholder pressures, and administrative practicality. Public Administration Review, 67 (2), 249-264.

Young, I. M. (1990). Justice and the politics of difference. Princeton: Princeton University Press.

Recibido: 05-02-2019

Aceptación de la versión final: 07-06-2019 